\title{
Application of Genetic Algorithm to the Shape Optimization of a Constrained Double-Chamber Muffler with Extended Tubes
}

\author{
Long-Jyi Yeh \\ Department of Mechanical Engineering, Tatung University, 40 Chungshan N. Rd., 3rd Sec., Taipei, Taiwan 104, R.O.C., \\ ljyeh@ttu.edu.tw \\ Ying-Chun Chang \\ Department of Mechanical Engineering, Tatung University, 40 Chungshan N. Rd., 3rd Sec., Taipei, Taiwan 104, R.O.C. \\ Min-Chie Chiu \\ Department of Mechanical Engineering, Tatung University, 40 Chungshan N. Rd., 3rd Sec., Taipei, Taiwan 104, R.O.C.
}

Follow this and additional works at: https://jmstt.ntou.edu.tw/journal

Part of the Mechanical Engineering Commons

\section{Recommended Citation}

Yeh, Long-Jyi; Chang, Ying-Chun; and Chiu, Min-Chie (2004) "Application of Genetic Algorithm to the Shape Optimization of a Constrained Double-Chamber Muffler with Extended Tubes," Journal of Marine Science and Technology: Vol. 12: Iss. 3, Article 8.

DOI: $10.51400 / 2709-6998.2238$

Available at: https://jmstt.ntou.edu.tw/journal/vol12/iss3/8

This Research Article is brought to you for free and open access by Journal of Marine Science and Technology. It has been accepted for inclusion in Journal of Marine Science and Technology by an authorized editor of Journal of Marine Science and Technology. 


\title{
APPLICATION OF GENETIC ALGORITHM TO THE SHAPE OPTIMIZATION OF A CONSTRAINED DOUBLE-CHAMBER MUFFLER WITH EXTENDED TUBES
}

\author{
Long-Jyi Yeh*, Ying-Chun Chang, and Min-Chie Chiu
}

\begin{abstract}
Key words: shape optimization, double-chamber muffler with extended tubes, transfer matrix method, space constraints, genetic algorithm.
\end{abstract}

\section{ABSTRACT}

As the compact design of a muffler system within a constrained environment of a existing machine room becomes obligatory, it also becomes essential to maximize the acoustic performance of mufflers under space constraints. In this paper, the shape optimization of a double-chamber muffler with an extended tube is presented. The main characteristic of the solution methodology is the use of genetic algorithm (GA) as the optimizer. In the paper, the acoustic performance of sound transmission loss (STL) derived by transfer matrix is conjugated with the techniques of GA searching. A numerical case of noise elimination in full band noise is also exemplified and fully discussed in this paper. Before GA operation, the presented theory of a single-chamber muffler with extended tubes is simulated and compared with Wang and Hsieh's experimental data for the purpose of accuracy check in the mathematical model. Thereafter, a simple optimal program in dealing with pure tone noise of $500 \mathrm{~Hz}$ has been pre-run to verify the correctness of the genetic algorithm before the design in full band noise will be performed. Results reflect the accuracy of the mathematical model and the correctness of the GA method. The optimal design of STL proposed in this study provides a quick and correct approach.

\section{INTRODUCTION}

Since the shape of a muffler needs to be compact for operation or maintenance purposes, there is an increasing interest in the field of acoustics in optimizing the sound transmission loss (STL) of a muffler under space constraints. Many researches of muffler designs have been well addressed. However, the discussion of optimal design under space constraints is rarely

Paper Submitted 04/22/04, Accepted 07/21/04. Author for Correspondence: Long-Jyi Yeh.E-mail: ljyeh@ttu.edu.tw.

*Department of Mechanical Engineering, Tatung University, 40 Chungshan N. Rd., $3^{\text {rd }}$ Sec., Taipei, Taiwan 104, R.O.C. emphasized. Bernhard [1] has introduced the shape optimization of simple expansion mufflers by using design sensitivity matrices. The space volume of the reactive silencer is non-constrained and the calculation of the design sensitivity matrices is difficult especially for complicated mufflers. In a previous work [9], the optimization of constrained single-chamber mufflers with side inlet/outlet by gradient method was discussed. A constrained double-chamber muffler with extended tubes was introduced to utilize the optimal design of the muffler. A GA optimizer and evolutionary search algorithms have been applied in this paper in order to solve the optimal design of mufflers. Here, the transfer matrix method $[6,7]$, which is based on the plane wave theory and used in the evaluation of $S T L$, is in conjunction with the GA method for the optimization work.

\section{THEORETICAL BACKGROUND}

\section{Sound transmission loss}

A 3-D cross-section view of a double-chamber muffler with extended tubes is shown in Figure 1. As shown in Figure 2, five segments of straight duct and four segments of extended duct are distinguished. Here, fourteen nodes representing the flowing situation within the muffler are built and exposed in Figure 3. The submatrices with respect to nine components of the muffler are expressed as follows:

$$
\begin{gathered}
\left(\begin{array}{c}
p_{1} \\
\rho_{o} c_{o} u_{1}
\end{array}\right)=e^{-j \frac{M_{1} k L_{12}}{1-M_{1}^{2}}}\left[\begin{array}{cc}
\cos \left(\frac{k L_{12}}{1-M_{1}^{2}}\right) & j \sin \left(\frac{k L_{12}}{1-M_{1}^{2}}\right) \\
j \sin \left(\frac{k L_{12}}{1-M_{1}^{2}}\right) & \cos \left(\frac{k L_{12}}{1-M_{1}^{2}}\right)
\end{array}\right] \\
\left(\begin{array}{c}
p_{2} \\
\rho_{o} c_{o} u_{2}
\end{array}\right)
\end{gathered}
$$




$$
\begin{aligned}
& \left(\begin{array}{c}
p_{2} \\
\rho_{o} c_{o} u_{2}
\end{array}\right)=\left[\begin{array}{ll}
T R 2_{1,1} & T R 2_{1,2} \\
T R 2_{2,1} & T R 2_{2,2}
\end{array}\right]\left(\begin{array}{c}
p_{4} \\
\rho_{o} c_{o} u_{4}
\end{array}\right) \\
& \left(\begin{array}{c}
p_{4} \\
\rho_{o} c_{o} u_{4}
\end{array}\right)=e^{-j \frac{M_{4} k L_{3}}{1-M_{4}^{2}}}\left[\begin{array}{ll}
\cos \left(\frac{k L_{3}}{1-M_{4}^{2}}\right) & j \sin \left(\frac{k L_{3}}{1-M_{4}^{2}}\right) \\
j \sin \left(\frac{k L_{3}}{1-M_{4}^{2}}\right) & \cos \left(\frac{k L_{3}}{1-M_{4}^{2}}\right)
\end{array}\right] \\
& \left(\begin{array}{c}
p_{5} \\
\rho_{o} c_{o} u_{5}
\end{array}\right) \\
& \left(\begin{array}{c}
p_{5} \\
\rho_{o} c_{o} u_{5}
\end{array}\right)=\left[\begin{array}{ll}
T R 4_{1,1} & T R 4_{1,2} \\
T R 4_{2,1} & T R 4_{2,2}
\end{array}\right]\left(\begin{array}{c}
p_{7} \\
\rho_{o} c_{o} u_{7}
\end{array}\right) \\
& \left(\begin{array}{c}
p_{7} \\
\rho_{0} c_{o} u_{7}
\end{array}\right)=e^{-j \frac{M_{7} k L_{456}}{1-M_{7}^{2}}}\left[\begin{array}{cc}
\cos \left(\frac{k L_{456}}{1-M_{7}^{2}}\right) & j \sin \left(\frac{k L_{456}}{1-M_{7}^{2}}\right) \\
j \sin \left(\frac{k L_{456}}{1-M_{7}^{2}}\right) & \cos \left(\frac{k L_{456}}{1-M_{7}^{2}}\right)
\end{array}\right] \\
& \left(\begin{array}{c}
p_{8} \\
\rho_{o} c_{o} u_{8}
\end{array}\right)
\end{aligned}
$$$$
\left(\begin{array}{c}
p_{8} \\
\rho_{o} c_{o} u_{8}
\end{array}\right)=\left[\begin{array}{ll}
T R 6_{1,1} & T R 6_{1,2} \\
T R 6_{2,1} & T R 6_{2,2}
\end{array}\right]\left(\begin{array}{c}
p_{10} \\
\rho_{o} c_{o} u_{10}
\end{array}\right)
$$$$
\left(\begin{array}{c}
p_{10} \\
\rho_{o} c_{o} u_{10}
\end{array}\right)=e^{-j \frac{M_{10} k L_{7}}{1-M_{10}^{2}}}\left[\begin{array}{ll}
\cos \left(\frac{k L_{7}}{1-M_{10}^{2}}\right) & j \sin \left(\frac{k L_{7}}{1-M_{10}^{2}}\right) \\
j \sin \left(\frac{k L_{7}}{1-M_{10}^{2}}\right) & \cos \left(\frac{k L_{7}}{1-M_{10}^{2}}\right)
\end{array}\right]
$$$$
\left(\begin{array}{c}
p_{11} \\
\rho_{o} c_{o} u_{11}
\end{array}\right)
$$$$
\left(\begin{array}{c}
p_{11} \\
\rho_{o} c_{o} u_{11}
\end{array}\right)=\left[\begin{array}{ll}
T R 8_{1,1} & T R 8_{1,2} \\
T R 8_{2,1} & T R 8_{2,2}
\end{array}\right]\left(\begin{array}{c}
p_{13} \\
\rho_{o} c_{o} u_{13}
\end{array}\right)
$$

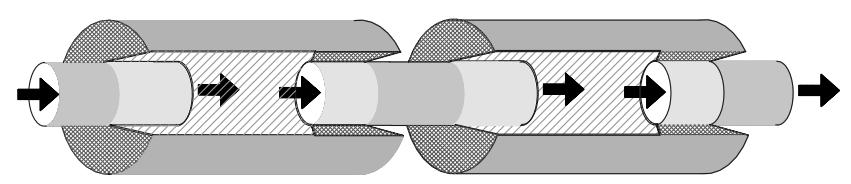

Fig. 1. 3-D cross-section of a double-chamber muffler with extended tubes.

$$
\begin{gathered}
\left(\begin{array}{c}
p_{13} \\
\rho_{o} c_{o} u_{13}
\end{array}\right)=e^{-j \frac{M_{13} k L_{89}}{1-M_{13}^{2}}}\left[\begin{array}{cc}
\cos \left(\frac{k L_{89}}{1-M_{13}^{2}}\right) & j \sin \left(\frac{k L_{89}}{1-M_{13}^{2}}\right) \\
j \sin \left(\frac{k L_{89}}{1-M_{13}^{2}}\right) & \cos \left(\frac{k L_{89}}{1-M_{13}^{2}}\right)
\end{array}\right] \\
\left(\begin{array}{c}
p_{14} \\
\rho_{o} c_{o} u_{14}
\end{array}\right)
\end{gathered}
$$

The detailed depiction of transfer matrix for straight duct and extended duct is demonstrated in APPENDICES A and B. By using the matrix substitution on equations (1)-(9), the system matrix of the muffler yields

$$
\left(\begin{array}{c}
p_{1} \\
\rho_{o} c_{o} u_{1}
\end{array}\right)=\left[\begin{array}{ll}
T_{1,1} & T_{1,2} \\
T_{2,1} & T_{2,2}
\end{array}\right]\left(\begin{array}{c}
p_{14} \\
\rho_{o} c_{o} u_{14}
\end{array}\right)
$$

The STL of a muffler is calculated as [6]

$$
\begin{aligned}
& S T L\left(f, Q, D_{2}, r t_{1}, r t_{2}, L_{3}, L_{5}, r t_{3}, r t_{4}, L_{9}\right) \\
& =20 \log \left(\frac{\left|T_{1,1}+T_{1,2}+T_{2,1}+T_{2,2}\right|}{2}\right)+10 \log \left(\frac{S_{1}}{S_{14}}\right)
\end{aligned}
$$

where $r t_{1}=\frac{L_{2}}{L_{3}} ; r t_{2}=\frac{L_{4}}{L_{3}} ; r t_{3}=\frac{L_{6}}{L_{7}} ; r t_{4}=\frac{L_{8}}{L_{7}}$;

$$
L_{7}=\frac{L_{o}-L_{1}-L_{5}-L_{9}-\left(r t_{1}+r t_{2}+1\right)}{1+r t_{3}+r t_{4}}
$$

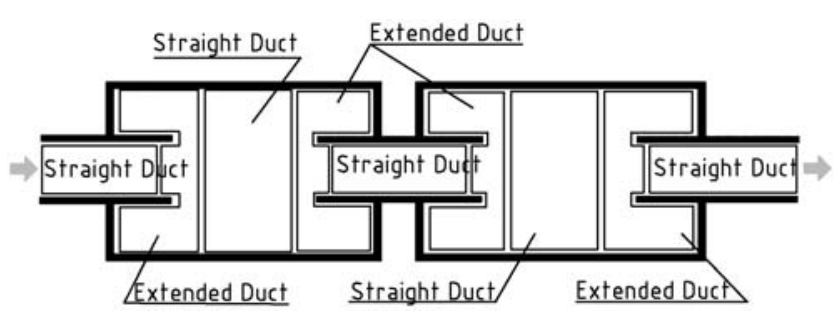

Fig. 2. Distinguish of muffler elements.

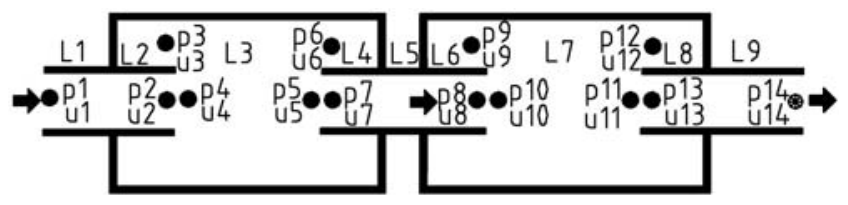

Fig. 3. Flow condition of a double-chamber muffler with extended tubes. 


\section{Overall sound pressure level}

Therefore, the silenced octave sound level at muffler outlet is

$$
S P L_{i}=S P L O_{i}-S T L_{i}
$$

Where (1) $S P L O_{i}$ is the original sound pressure level $(S T L)$ at inlet of muffler (or pipe outlet), and $i$ is the index of octave band frequency.

(2) $S T L_{i}$ is the muffler's $S T L$ with respect to the relative octave band frequency.

(3) $S P L_{i}$ is the silenced $S P L$ at outlet of muffler with respect to the relative octave band frequency outlet is

Finally, the overall $S P L_{T}$ silenced by muffler at

$$
\begin{aligned}
& S P L_{T}=10 \times \log \left\{\sum_{i=1}^{8} 10^{S P L_{i} / 10}\right\} \\
& =10 \times \log \left\{\begin{array}{cc}
{[S P L O(f=63)-} & {[S P L O(f=125)-} \\
+10^{S T L(f=63)] / 10}+10^{S T L(f=125)] / 10} \\
{[S P L O(f=250)-} & {[S P L O(f=500)-} \\
+10^{S T L(f=250)] / 10}+10^{S T L(f=500)] / 10} \\
{[S P L O(f=1000)-} & {[S P L O(f=2000)-} \\
+10^{S T L(f=1000)] / 10}+10^{S T L(f=2000)] / 10} \\
{[S P L O(f=4000)-} & {[S P L O(f=8000)-} \\
+10^{S T L(f=4000)] / 10}+10^{S T L(f=8000)] / 10}
\end{array}\right\}
\end{aligned}
$$

\section{Objective function}

By using the formula of Eq. (13), the objective function used in GA optimization with respect to muffler is

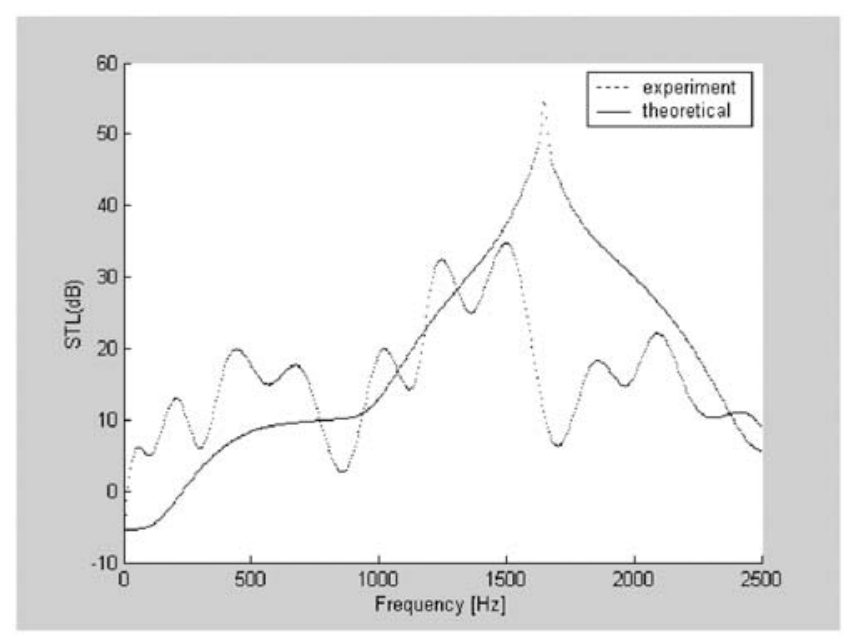

Fig. 4. Performance of a single-chamber muffler with extended tubes at the mean flow velocity of $3.4 \mathrm{~m} / \mathrm{s}\left[D_{1}=D_{2}=0.0365(\mathrm{~m}) ; D_{o}=\right.$ $\left.0.108(\mathrm{~m}) ; L_{1}=L_{5}=0.1(\mathrm{~m}) ; L_{2}=L_{4}=0.052(\mathrm{~m}) ; L_{3}=0.104(\mathrm{~m})\right]$.

$$
Q B J=S T L\left(f, Q, D_{2}, r t_{1}, r t_{2}, L_{3}, L_{5}, r t_{3}, r t_{4}, L_{9}\right)
$$

\section{MODEL CHECK}

Before performing GA optimal simulation on mufflers, the accuracy check of mathematical model on the fundamental element of a single-chamber muffler with extended tubes was performed by experimental data [8]. As depicted in Figure 4, the accuracy comparison between theoretical and experimental data for the model is in a good agreement. Therefore, the proposed fundamental mathematical model is acceptable. Consequently, the developed model of double-chamber muffler with extended tubes linked with numerical method is applied for the shape optimization in the following section.

\section{GENETIC ALGORITHM}

The concept of Genetic Algorithms, first formalized by Holland [3] and extended to functional optimization by Jong [5] later involves the use of optimization search strategies patterned after Darwinian notion of natural selection and evolution. Being a stochastic search technique, the GA's differ from deterministic search methods in the way the search vector is calculated. GA's start the optimal search from a population. Each individual in the population represents a point in the search space for which the dimension is determined by the number of design variable. The real valued design variables are encoded into finite length binary strings (i.e. chromosomes). For each generation, the genetic search calculates the fitness of every individual in the population. While all chromosomes are eligible to survive to form the mating pool for the next generation, the selective pressure gives the better members a higher chance. Once the selection is complete, a second operator, crossover, performs genetic code exchange between parents randomly chosen among the survivors. The offspring constitute the new generation. A background operator, mutation, also plays a role in determining the final state. In addition, to reserve the best gene as the parent generation for next generation, a conservative operator, elitism, is performed, accordingly. The search continues until a stopping criteria (preset maximal generation) is reached. A brief description of operator in the genetic algorithm is categorized as follows:

A. Populations and Chromosomes. For the optimization (minimum) of the objective function $(O B J)$, the design parameters are $\left(x_{1}, x_{2}, \ldots x_{k}\right)$. As the bit length of bit_n, which in terms of the chromosome, was chosen first, the interval of the 
$k^{\text {th }}$ design parameter with $\left[L_{b}, U_{b}\right]_{k}$ was thereafter mapped to the band of binary value. The mapping system between the variable interval of $\left[L_{b}, U_{b}\right]_{k}$ and the $k^{\text {th }}$ binary chromosome of $[000000 \ldots 0000$ $\sim 111111 \ldots 1111]_{k}$ was then built. The encoding from $x$ to $B 2 D$ can be performed as

$$
B 2 D_{k}=\text { integer }\left\{\frac{x_{k}-L b_{k}}{U b_{k}-L b_{k}}\left(2^{b i t \_n}-1\right)\right\}
$$

The initial population was built up by randomization. The parameter set was encoded to form a string which represented the chromosome. The more there was an increase in bit length, the better resolution of optimization.

By evaluation of the objective function $(O B J)$, the whole set chromosome of $\left[B 2 D_{1}, B 2 D_{1}, \ldots, B 2 D_{k}\right]$ that changed from binary form to decimal form was then assigned a fitness by decoding the transformation system individually.

fitness $=O B J\left(X_{1}, X_{2}, \ldots, X_{k}\right)$;

Where $X_{k}=B 2 D_{k}^{*}\left(U b_{k}-L b_{k}\right) /\left(2^{b i t_{-} n}-1\right)+L b_{k}$

B. Parents. By using the probabilistic computation weighted by the relative fitness, pairs of chromosome were selected as parents. The weighted roulette wheel selection was then applied. Each individual in the population was assigned space on the roulette wheel which was proportional to the individual relative fitness. For $n$ set of parent in the mating pool, the weighted roulette wheel for the $k k^{\text {th }}$ individual was represented as $\frac{\text { fintess }_{k k}}{\sum_{i=1}^{n} \text { fintess }_{i}} \times 100 \%$. Individuals with
$\Delta P=0.0104 u^{1.9} D_{H}^{-1.23} L$

the largest portion on the wheel have the greatest probability to be selected as parent generation for

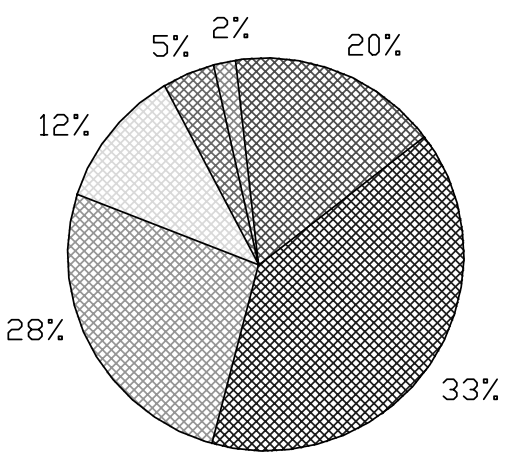

Fig. 5. Weighted roulette wheel method of selection. the next generation. A typical selection scheme, a weighted roulette wheel is depicted in Figure 5.

C. Crossover. One pair of offspring was generated from the selected parent by crossover. Crossover occurred with a probability of $p c$. Both the random selection of a crossover and combination of the two parent's genetic data were then preceded. The scheme of single-point crossover was chosen in GA's optimization. Recombination and parent selection were the methods for the evolution in GA. For the k numbers of design variables in the objective function $(O B J)$, the crossover operation with respect to individual chromosome of $\left[B 2 D_{1}\right.$, $\left.B 2 D_{2}, \ldots, B 2 D_{k}\right]$ was carried out in the binary form simultaneously. A typical scheme of single-point crossover is depicted in Figure 6.

D. Mutation. Genetically, mutation occurred with a probability of pm of which the new and unexpected points were brought into the GA optimizer's search domain. The operation was performed by changing the value of the bit from one to zero or zero to one at the selected point. It was an essential operation to increase the diversity of population that was able to escape from the local optimum during GA's optimization. For the $k$ numbers of design variables in the objective function $(O B J)$, the mutation operation with respect to individual chromosome of $\left[B 2 D_{1}, B 2 D_{2}, \ldots, B 2 D_{k}\right]$ was carried out in the binary form simultaneously. A typical scheme of mutation is depicted in Figure 7. E. Elitism. To prevent the best gene from the disappearing and improve the accuracy of optimization during reproduction, the elitism scheme of keeping the best gene (one pair) in the parent generations was thus developed and presented.

F. New Generation. Reproduction includes selection, crossover, mutation and elitism. The reduplication continued until a new generation was constructed and the original generation was substituted. Highly fit characteristics produced

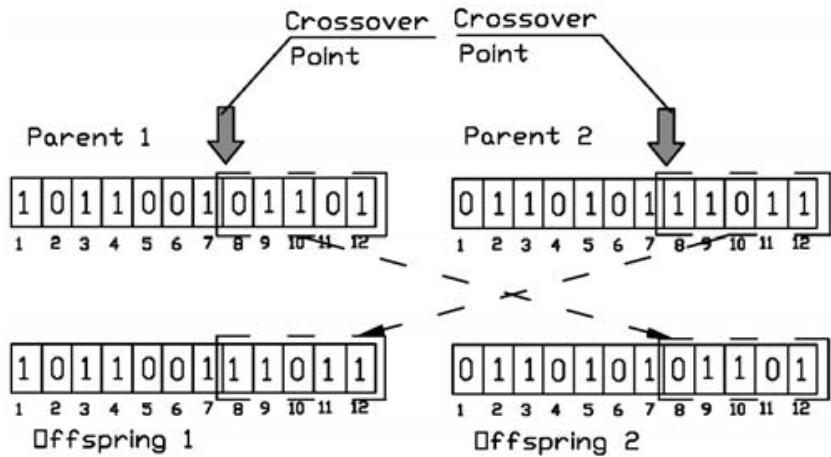

Fig. 6. Scheme of single-point crossover. 
more copies of themselves in subsequent generation resulting in a movement of the population towards an optimal direction. The process was terminated when the number of generation exceeded a pre-selected value of $N_{g}$.

The operations in GA method are pictured in Figure 8. In addition, the block diagram of GA optimization on mufflers is depicted in Figure 9.

\section{GA OPTIMIZATION}

\section{Case study}

A noise control of diesel engine at the exhausted outlet shown in Figure 10 is introduced as the numerical case. The spectrum of equipment's SPL at the pipe outlet (the muffler inlet) is shown below,

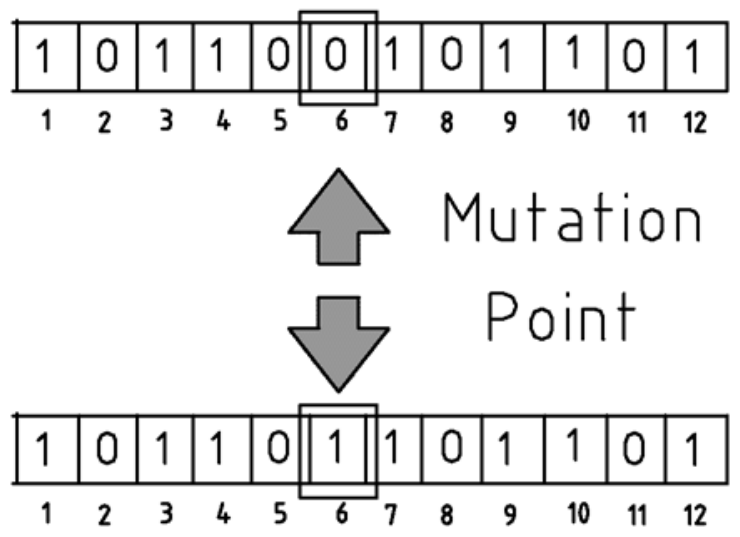

Fig. 7. Scheme of mutation.

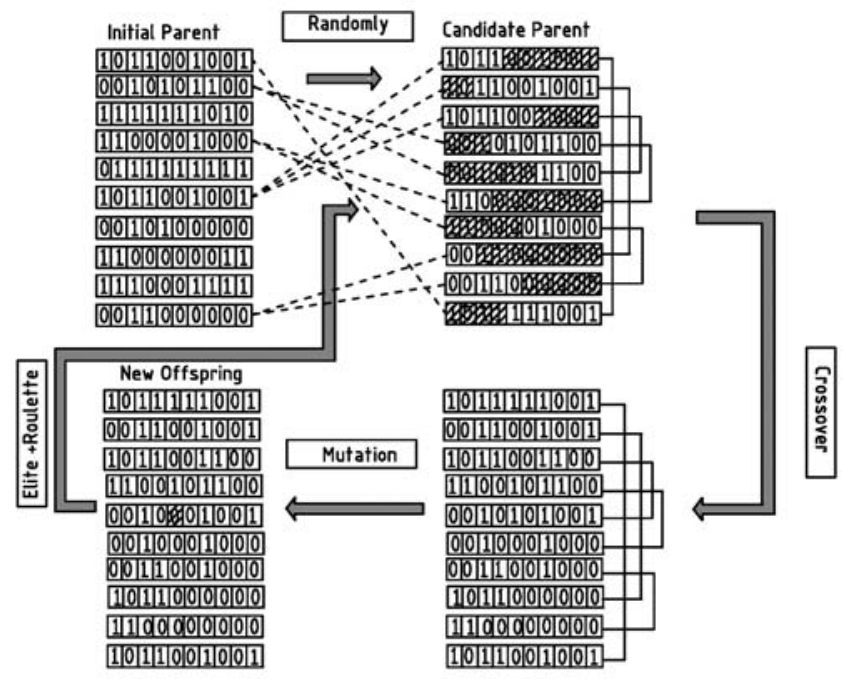

Fig. 8. Procedure and technique of GA operators.

$\begin{array}{cccccccccc}\mathrm{F}(\mathrm{Hz}) & 31.5 & 63 & 125 & 250 & 500 & 1 \mathrm{k} & 2 \mathrm{k} & 4 \mathrm{k} & 8 \mathrm{k} \\ \mathrm{SPL}-\mathrm{dB}(\mathrm{A}) & 86 & 90 & 94 & 93 & 104 & 95 & 91 & 88 & 64\end{array}$

The available space for the muffler is $0.3^{\mathrm{M}} \mathrm{L} * 0.3$ ${ }^{\mathrm{M}} \mathrm{W} * 1.0^{\mathrm{M}} \mathrm{H}$. By equation (13), nine design parameters are chosen in GA optimization. According to Bies and Hansen [2], the pressure drop $(\Delta P)$ of ducts is estimated

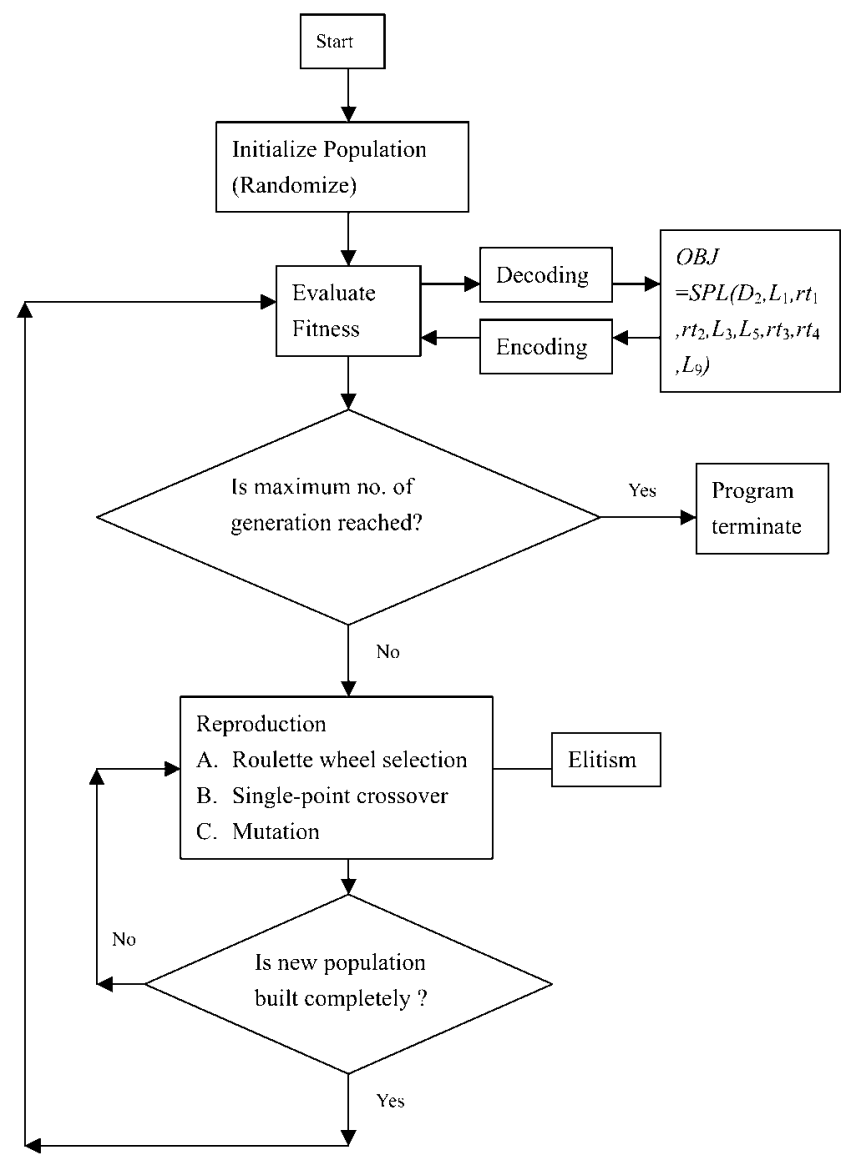

Fig. 9. Block diagram of GA optimization.

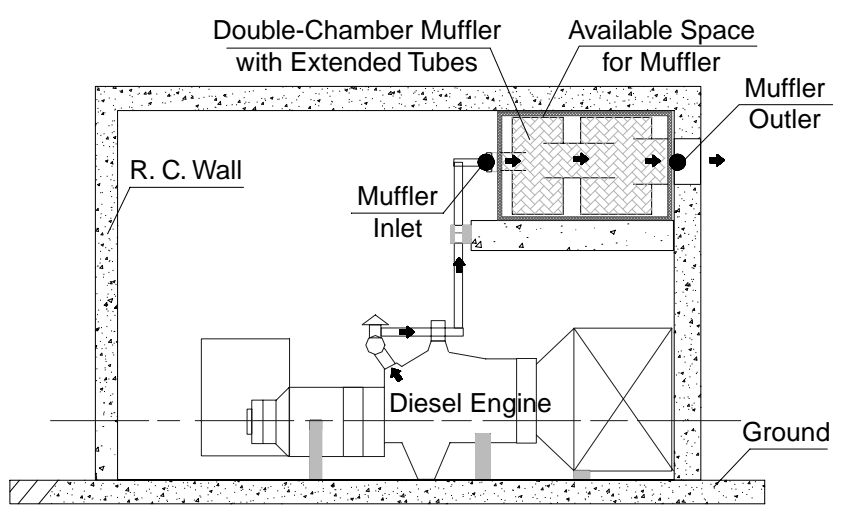

Fig. 10. Elevation plan of the noise control system inside a diesel engine room. 
as

$$
\Delta P=0.0104 u^{1.9} D_{H}^{-1.23} L
$$

where $u$ is the flow velocity, $D_{H}$ is the fluid diameter and $L$ is the length of duct. To diminish the effect of pressure drop in the muffler, the necessities of minimal diameters at both ends specified to no less than 0.0762 (m) (commercial standard pipe dimension) are demanded. A series of assumptions of the constrained condition in design work is illustrated as

$0.0762(\mathrm{~m}) \leq D_{2} \leq 0.5(\mathrm{~m}) ; 0.1(\mathrm{~m}) \leq L_{1} \leq 0.2(\mathrm{~m}) ; 0.1$ $\leq r t_{1} \leq 1.0 ; 0.1 \leq r t_{2} \leq 1.0 ; 0.1(\mathrm{~m}) \leq L_{3} \leq 1.5(\mathrm{~m}) ; 0.1(\mathrm{~m})$ $\leq L_{5} \leq 0.3(\mathrm{~m}) ; 0.1 \leq r t_{3} \leq 1.0 ; 0.1 \leq r t_{4} \leq 1.0 ; 0.1(\mathrm{~m}) \leq$ $L_{9} \leq 0.2(\mathrm{~m})$

The space constraints for the muffler are shown in Figure 11 , and the design volume flow rate $(Q)$ is confined to $0.8\left(\mathrm{~m}^{3} / \mathrm{s}\right)$.

\section{Correctness on GA}

Before the muffler design in full band noise reduction being performed, a simple optimal program dealing with pure tone noise of $500 \mathrm{~Hz}$ has been pre-run to verify the correctness of results obtained from genetic optimization. The optimization system is programmed by MATLAB and run in IBM PC - Pentium IV. In order to hasten the convergence in GA's solution, the larger number of population $\left(N_{p}\right)$, generation $\left(N_{g}\right)$ and bit length $\left(N_{b}\right)$ are set to 60,500 and 40 , respectively.
According to Johnson and Yahya [4], both the typical ratio crossover $(p c)$ and mutation ratio $(p m)$ used in following GA optimization are chosen as 0.8 and 0.05 independently. The optimal results are listed and plotted in Table 1 and Figure 12, respectively. As the results shown in Figure 12 indicate that the STL of the doublechamber muffler with extended tubes is maximized at the desired frequency of $500 \mathrm{~Hz}$. Consequently, the correctness by using GA optimization technique is verified.

\section{RESULTS AND DISCUSSION}

\section{Results}

As described above, the larger number of population $\left(N_{p}\right)$, generation $\left(N_{g}\right)$ and bit length $\left(N_{b}\right)$ are also set to 60,500 , and 40 individually. To identify the effects among GA operators, four trial cases with different values of control parameters ( $p c, p m$ and elit) are thus varied and discussed. The elitism (elt) is an optional item in which the flag of elt is set to " 1 " for the selection and " 0 " for discard of elitism' scheme. Four cases of GA parameters are adopted and proceeded as:

case 1: $p c=0.8 ; p m=0.05 ;$ elt $=1$ [crossover, mutation and elitism are selected]

case $2: p c=0.8 ; p m=0.05 ;$ elt $=0 \quad$ [crossover and mutation are selected]

case 3: $p c=0.8 ; p m=0 ;$ elt $=1$ [crossover and elitism are selected]

case $4: p c=0 ; p m=0.05 ;$ elt $=1$ [mutation and elitism

Table 1. Optimal results for GA test at pure tone of $500 \mathrm{~Hz}$

\begin{tabular}{cccccccccccccccccc}
\hline \multicolumn{1}{ll}{ GA parameters } & \multicolumn{1}{c}{ Results } & & & $\begin{array}{c}\text { Elapsed } \\
\text { time }\end{array}$ \\
\hline$p c$ & $p m$ & elt & $D_{2}(\mathrm{~m})$ & $L_{1}(\mathrm{~m})$ & $r t_{1}$ & $r t_{2}$ & $L_{3}(\mathrm{~m})$ & $L_{5}(\mathrm{~m})$ & $r t_{3}$ & $r t_{4}$ & $L_{9}(\mathrm{~m})$ & $S T L$ & $t(\mathrm{Min})$. \\
0.8 & 0.05 & 1 & 0.0861 & 0.1881 & 0.5245 & 0.5157 & 0.3291 & 0.2452 & 0.5197 & 0.6627 & 0.1982 & 288.5 & 2.28 \\
\hline
\end{tabular}

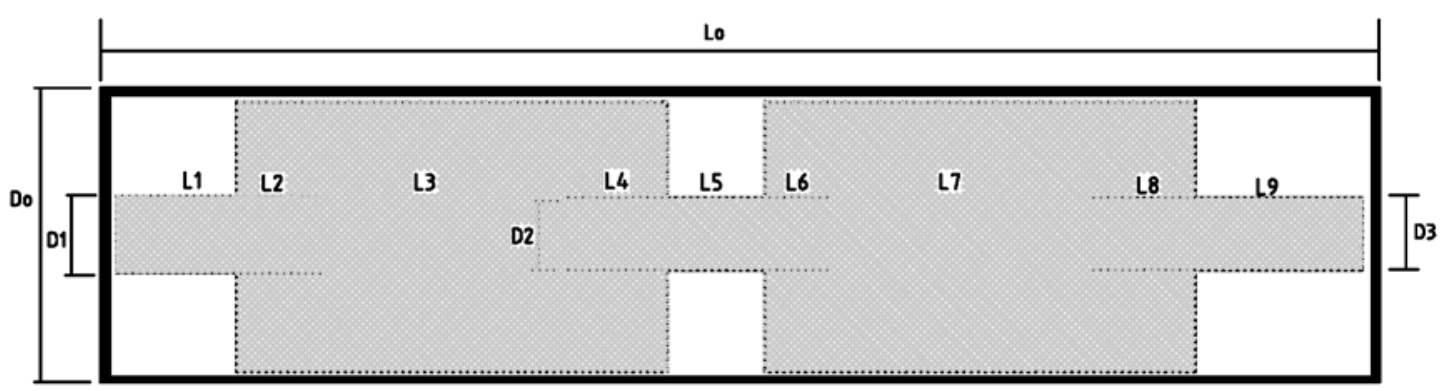

Fig. 11. Space constraints of a double-chamber muffler with extended tubes $\left[L_{o}=1.0 \mathrm{~m} ; D_{o}=0.3 \mathrm{~m}\right]$. 
Table 2. Comparison of results for the variations of GA parameters

\begin{tabular}{|c|c|c|c|c|c|c|c|c|c|c|c|c|c|c|}
\hline & \multicolumn{7}{|c|}{ GA parameters } & \multicolumn{6}{|l|}{ Results } & \multirow{2}{*}{$\begin{array}{c}\begin{array}{c}\text { Elapsed } \\
\text { time }\end{array} \\
t \text { (Min.) }\end{array}$} \\
\hline & $p c$ & $p m$ & elt & $D_{2}(\mathrm{~m})$ & $L_{1}(\mathrm{~m})$ & $r t_{1}$ & $r t_{2}$ & $L_{3}(\mathrm{~m})$ & $L_{5}(\mathrm{~m})$ & $r t_{3}$ & $r t_{4}$ & $L_{9}(\mathrm{~m})$ & $S T L$ & \\
\hline Case 1 & 0.8 & 0.05 & 1 & 0.2998 & 0.0505 & 0.6587 & 0.1019 & 0.2007 & 0.1231 & 0.9931 & 0.3494 & 0.0503 & 77.9 & 11.75 \\
\hline Case2 & 0.8 & 0.05 & 0 & 0.2996 & 0.0637 & 0.9032 & 0.1012 & 0.2112 & 0.0986 & 0.9701 & 0.6617 & 0.0571 & 78.8 & 11.70 \\
\hline Case 3 & 0.8 & 0 & 1 & 0.2851 & 0.1084 & 0.9006 & 0.1143 & 0.2005 & 0.1021 & 0.5301 & 0.2514 & 0.0790 & 80.4 & 11.68 \\
\hline Case4 & 0 & 0.05 & 1 & 0.0805 & 0.0788 & 0.1699 & 0.1395 & 0.1568 & 0.0664 & 0.2067 & 0.1032 & 0.0665 & 78.0 & 11.53 \\
\hline
\end{tabular}

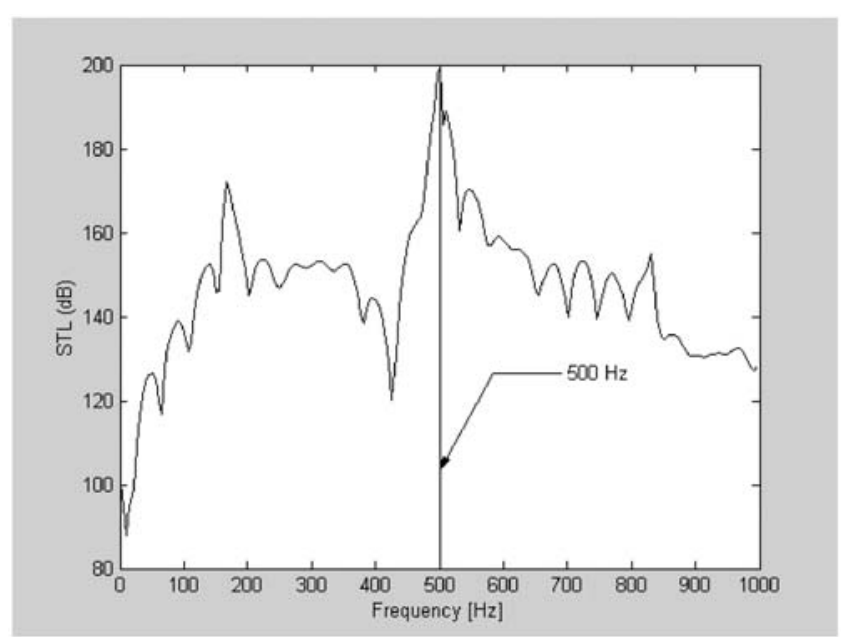

Fig. 12. Optimal STL for GA test at pure tone of $500 \mathrm{~Hz}[p c=0.8 ; p m$ $=0.05 ;$ elt $=1]$.

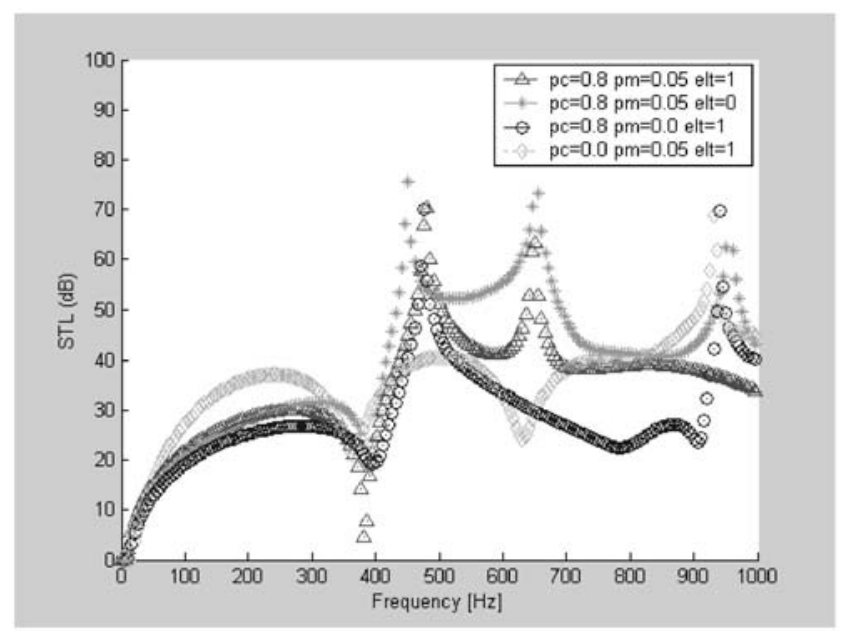

Fig. 13. Optimal STL for full band noise at four sets of GA's parameters.

are selected]

The results for the four cases are summarized in Table 2 .

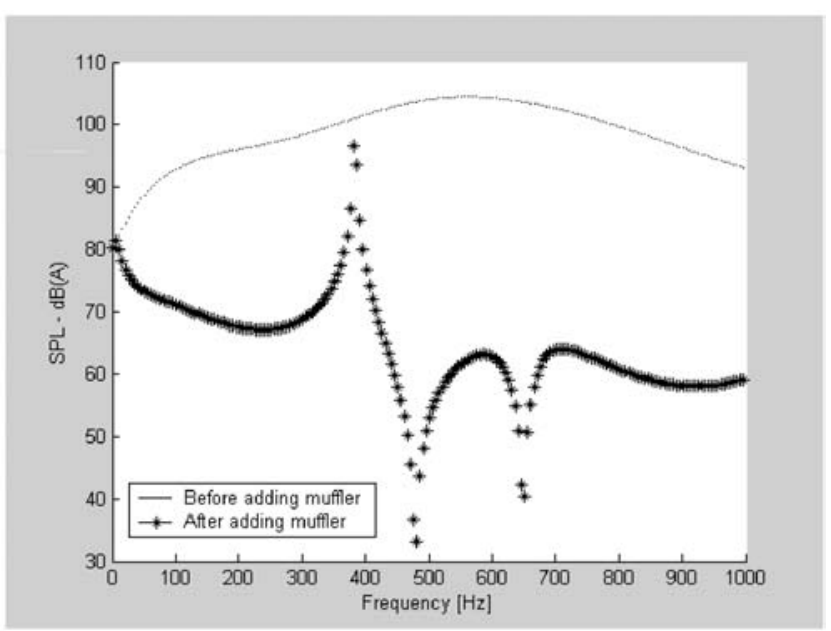

Fig. 14. Muffler's performance for full band noise at $p c=0.8, p m=$ 0.05 and $e l t=1$.

\section{Discussion}

As illustrated in Table 2, the first case in which crossover, mutation and elitism all applied has the minimal value of SPL compared with other cases. The performance curves of STL with respect to four cases are plotted in Figure 13. By adopting the design data from case 1 , a performance curve of the muffler at exhausted outlet before and after muffler being added is shown in Figure 14. It is observed that the noise reduction of muffler over the full band of spectrum is obvious. Therefore, the optimal design of muffler is achieved.

\section{CONCLUSIONS}

It has been shown that GA can be used in the optimization of muffler design by adjusting the shape of the muffler under space constraints. Since there is no need of complicated calculations in the mathematical derivative and in the good selection in the starting point, GA becomes easier to use compared to the gradient 
optimization method. The case study reveals that the GA parameters including crossover, mutation and elitism must be taken into consideration in order to achieve the accurate optimization of the GA. As the characteristic of rapidity and correctness presented in this study, the novel scheme of GA optimization becomes reliable and applicable in muffler design under space constraints with the presentation of its rapidity and correctness characteristics.

\section{NOMENCLATURE}

This paper is constructed on the basis of the following notations.

\begin{tabular}{|c|c|}
\hline$c_{o}$ & sound speed $\left(\mathrm{m} \mathrm{s}^{-1}\right)$ \\
\hline$D_{o}$ & $\begin{array}{l}\text { diameter's constraint of muffler } \\
(\mathrm{m})\end{array}$ \\
\hline$D_{1}, D_{2}, D_{3}$ & $\begin{array}{l}\text { diameters of muffler's compo- } \\
\text { nents }(\mathrm{m})\end{array}$ \\
\hline elt & $\begin{array}{l}\text { selection of elite ( } 1 \text { for yes and } 0 \\
\text { for no) }\end{array}$ \\
\hline$j$ & $\sqrt{-1}$ \\
\hline$k$ & wave number \\
\hline$k_{c}$ & stagnation pressure loss factor \\
\hline$L_{o}$ & length constraint of muffler $(\mathrm{m})$ \\
\hline$L_{1}, L_{2}, L_{3}, L_{4}, L_{5}$, & lengths of muffler's \\
\hline$L_{6}, L_{7}, L_{8}, L_{9}$ & components $(\mathrm{m})$ \\
\hline$L_{12}$ & $L_{1}+L_{2}(\mathrm{~m})$ \\
\hline$L_{456}$ & $L_{4}+L_{5}+L_{6}(\mathrm{~m})$ \\
\hline$L_{89}$ & $L_{8}+L_{9}(\mathrm{~m})$ \\
\hline$M_{i}$ & $\underset{i}{\text { mean flow Mach number at point }}$ \\
\hline$N_{b}$ & bit length \\
\hline$N_{g}$ & maximum number of generation \\
\hline$N_{p}$ & number of population \\
\hline$p c$ & crossover ratio \\
\hline$p_{c, i}$ & $\begin{array}{l}\text { aeroacoustic pressure at point } i \\
(\mathrm{~Pa})\end{array}$ \\
\hline$p_{i}$ & $\begin{array}{l}\text { pressure; acoustic pressure at } \\
\text { point } i(\mathrm{~Pa})\end{array}$ \\
\hline pm & mutation ratio \\
\hline$Q$ & venting volume flow rate $\left(\mathrm{m}^{3} / \mathrm{s}\right)$ \\
\hline $\bar{S}_{i}$ & section area at point $i\left(\mathrm{~m}^{2}\right)$ \\
\hline$S T L$ & sound transmission loss $(\mathrm{dB})$ \\
\hline$S P L$ & sound pressure level - $\mathrm{dB}(\mathrm{A})$ \\
\hline$u_{i}$ & $\begin{array}{l}\text { acoustic particle velocity at point } \\
\qquad i\left(\mathrm{~m} \mathrm{~s}^{-1}\right)\end{array}$ \\
\hline$v_{c, i}$ & $\begin{array}{l}\text { aeroacoustic mass velocity at } \\
\text { point } i\left(\mathrm{~kg} \mathrm{~s}^{-1}\right)\end{array}$ \\
\hline$V_{i}$ & $\begin{array}{l}\text { mean flow velocity at point } i \\
\left(\mathrm{~m} \mathrm{~s}^{-1}\right)\end{array}$ \\
\hline$Y_{i}$ & $\begin{array}{l}\text { characteristic impedance at point } \\
\quad i\end{array}$ \\
\hline$\rho_{o}$ & air density $\left(\mathrm{kg} \mathrm{m}^{-3}\right)$ \\
\hline
\end{tabular}

\section{REFERENCES}

1. Bernhard, R.J., "Shape Optimization of Reactive Mufflers," Noise Control Engin. J., Vol. 27, No. 1, pp. 10-17 (1986).

2. Bie, D.A. and Hansen, C. H., Engineering Noise Control, Unwin Hyman, UK (1988).

3. Holland, J.H., Adaptation in Natural and Artificial System, The University of Michigan Press, Ann Arbor (1975).

4. Johnson, J.M. and Yahya, R.S., "Genetic Algorithm Optimization and Its Application to Antenna Design," Proceedings of the IEEE Antennas and Propagation Society International Symposium, pp. 326-331 (1994).

5. Jong, D., "Analysis of the Behavior of a Class of a Genetic Adaptive Systems," Ph.D. Dissertation, The University of Michigan Press, Ann Arbor (1975).

6. Munjal, M.L., Acoustics of Ducts and Mufflers with Application to Exhaust and Ventilation System Design, John Wiley and Sons, New York (1987).

7. Sathyanarayana, Y. and Munjal, M.L., "A Hybrid Approach for Aeroacoustic Analysis of the Engine Exhaust System," Appl. Acoustics, Vol. 60, pp. 425-450 (2000).

8. Wang C.N. and Hsieh, C.C., "Experimental Study for Muffler Components with Flow," Bull. Coll. Engin., N. T.U., Vol. 78, pp. 67-74 (2000).

9. Yeh, L.J., Chang, Y.C., Chiu, M.C., and Lai, G.J., "Computer-Aided Optimal Design of A Single-Chamber Muffler with Side Inlet/Outlet Under Space Constraints," J. Marine Sci. Technol., Vol. 11, No. 4, pp. 1-8 (2003).

\section{APPENDIX A}

\section{Transfer Matrix of Straight Duct}

For a three dimensional wave with moving medium, three kinds of governing equations are

I. Mass continuity equation:

$$
\rho_{o} \nabla \cdot \vec{u}+\frac{D \rho}{D t}=0
$$

II. Momentum equation :

$$
\rho_{o} \frac{D \vec{u}}{D t}+\nabla p=0
$$

III. Energy equation (isentropic):

$$
\left(\frac{\partial p}{\partial \rho}\right)_{s}=\frac{\gamma\left(p_{o}+p\right)}{\rho_{o}+\rho} \approx \frac{\gamma \rho_{o}}{\rho_{o}}=c_{o}^{2} \text { or } \frac{p}{\rho}=c_{o}^{2}
$$


By partial derivation and substitution in Eqs. (A1), (A2), and (A3), the wave governing equation yields

$$
\left(\frac{D^{2}}{D t^{2}}-c_{o}^{2} \nabla^{2}\right) p=0
$$

where $\frac{D}{D t}=\frac{\partial}{\partial t}+\vec{V}_{o} \cdot \nabla$ is the total derivative. $\vec{V}_{o}$ is the mean velocity

For cylindrical polar coordinate system (for cylindrical tubes), the Laplacian $\nabla^{2}$ is

$$
\nabla^{2}=\frac{\partial^{2}}{\partial r^{2}}+\frac{1}{r} \frac{\partial}{\partial r}+\frac{1}{r^{2}} \frac{\partial^{2}}{\partial \theta^{2}}+\frac{\partial^{2}}{\partial z^{2}}
$$

By using separation of variables method in Eqs. (A4) and (A5), it yields

$$
\begin{aligned}
& p(r, \theta, z, t)=\sum_{m=0}^{\infty} \sum_{n=0}^{\infty} J_{m}\left(k_{r, m, n} r\right) \\
& \left(C_{1, m, n} e^{-j k_{z, m, n}^{+}}+C_{2, m, n} e^{+j k_{z, m, n^{z}}^{-}}\right) e^{j w t} \\
& u_{Z}(r, \theta, z, t) \\
& =\frac{1}{\rho_{o} c_{o}} \sum_{m=0}^{\infty} \sum_{n=0}^{\infty} J_{m}\left(k_{r, m, n} r\right) e^{j w \theta} e^{j w t} \\
& \left(\begin{array}{c}
\frac{k_{z, m, n}^{+}}{k_{o}-M k_{z, m, n}^{+}} C_{1, m, n} e^{-j k_{z, m, n}^{+} z} \\
+\frac{k_{z, m, n}^{+}}{k_{o}-M k_{z, m, n}^{+}} C_{2, m, n} e^{+j k_{z, m, n}^{-}} \\
k_{z, m, n}^{ \pm}=\frac{\mp M_{1} k_{o}+\left[k_{o}^{2}-\left(1-M_{1}^{2}\right) k_{r, m, n}^{2}\right]^{1 / 2}}{1-M_{1}^{2}}
\end{array}\right.
\end{aligned}
$$

For the fundamental mode of $(m=0, n=0)$, only a plane wave would propagate if the frequencies of $f$ are smaller than both of the diametral cut-off frequency $-f_{c 1}$ and the axisymmetric radial cut-off frequency $-f_{c 2}$.

where $f_{c 1}=\frac{1.84 c_{o}}{\pi D}\left(1-M_{1}^{2}\right)^{1 / 2} ; f_{c 1}=\frac{3.83 c_{o}}{\pi D}\left(1-M_{1}^{2}\right)^{1 / 2}$

For one dimensional wave propagating in a symmetric straight duct, the acoustic pressure and particle velocity are reduced as

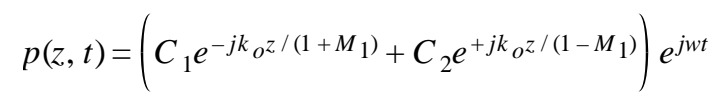

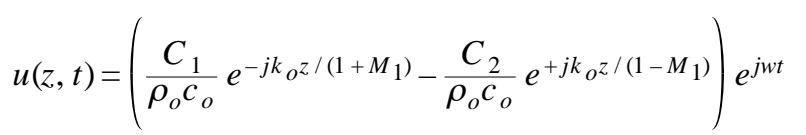

By taking boundary conditions of pt $1(z=0)$ and pt $2\left(z=L_{12}\right)$ into Eqs. (A10) (A11), it yields

$$
\begin{aligned}
& \left(\begin{array}{c}
p_{1} \\
\rho_{o} c_{o} u_{1}
\end{array}\right)=\left[\begin{array}{cc}
1 & 1 \\
1 & -1
\end{array}\right]\left(\begin{array}{l}
C_{1} \\
C_{2}
\end{array}\right) \\
& \left(\begin{array}{c}
p_{2} \\
\rho_{o} c_{o} u_{2}
\end{array}\right)=\left[\begin{array}{cc}
e^{-j k+L_{12}} & e^{+j k^{-} L_{12}} \\
e^{-j k+L_{12}} & -e^{+j k^{-} L_{12}}
\end{array}\right]\left(\begin{array}{l}
C_{1} \\
C_{2}
\end{array}\right)
\end{aligned}
$$

where $k^{+}=\frac{k_{o}}{1+M_{1}} ; k^{-}=\frac{k_{o}}{1-M_{1}}$

Combination of Eqs. (A12) and (A13) carries out that

$$
\begin{gathered}
\left(\begin{array}{c}
p_{1} \\
\rho_{o} c_{o} u_{1}
\end{array}\right)=e^{-j \frac{M_{1} k L_{12}}{1-M_{1}^{2}}}\left[\begin{array}{cc}
\cos \left(\frac{k L_{12}}{1-M_{1}^{2}}\right) & j \sin \left(\frac{k L_{12}}{1-M_{1}^{2}}\right) \\
j \sin \left(\frac{k L_{12}}{1-M_{1}^{2}}\right) & \cos \left(\frac{k L_{12}}{1-M_{1}^{2}}\right)
\end{array}\right] \\
\left(\begin{array}{c}
p_{2} \\
\rho_{o} c_{o} u_{2}
\end{array}\right)
\end{gathered}
$$

As the derivation in Eq. (A13), the four-pole matrix between pt $4(z=0)$ and pt $5\left(z=L_{3}\right)$ with mean flow is expressed in equation (A15).

$$
\begin{aligned}
& \left(\begin{array}{c}
p_{4} \\
\rho_{o} c_{o} u_{4}
\end{array}\right)=e^{-j \frac{M_{4} k L_{3}}{1-M_{4}^{2}}}\left[\begin{array}{ll}
\cos \left(\frac{k L_{3}}{1-M_{4}^{2}}\right) & j \sin \left(\frac{k L_{3}}{1-M_{4}^{2}}\right) \\
j \sin \left(\frac{k L_{3}}{1-M_{4}^{2}}\right) & \cos \left(\frac{k L_{3}}{1-M_{4}^{2}}\right)
\end{array}\right] \\
& \left(\begin{array}{c}
p_{5} \\
\rho_{o} c_{o} u_{5}
\end{array}\right)
\end{aligned}
$$

Thus, the four-pole matrix between pt $7(z=0)$ and pt $8\left(z=L_{456}\right)$ with mean flow is expressed in equation (A16).

$$
\left(\begin{array}{c}
p_{7} \\
\rho_{o} c_{o} u_{7}
\end{array}\right)=e^{-j \frac{M_{7} k L_{456}}{1-M_{7}^{2}}}\left[\begin{array}{cc}
\cos \left(\frac{k L_{456}}{1-M_{7}^{2}}\right) & j \sin \left(\frac{k L_{456}}{1-M_{7}^{2}}\right) \\
j \sin \left(\frac{k L_{456}}{1-M_{7}^{2}}\right) & \cos \left(\frac{k L_{456}}{1-M_{7}^{2}}\right)
\end{array}\right]
$$




$$
\left(\begin{array}{c}
p_{8} \\
\rho_{o} c_{o} u_{8}
\end{array}\right)
$$

By the same reason, the four-pole matrix between pt $10(z=0)$ and pt $11\left(z=L_{7}\right)$ with mean flow is expressed in equation (A17).

$$
\left(\begin{array}{c}
p_{10} \\
\rho_{o} c_{o} u_{10}
\end{array}\right)=e^{-j \frac{M_{10} k L_{7}}{1-M_{10}^{2}}}\left[\begin{array}{cc}
\cos \left(\frac{k L_{7}}{1-M_{10}^{2}}\right) & j \sin \left(\frac{k L_{7}}{1-M_{10}^{2}}\right) \\
j \sin \left(\frac{k L_{7}}{1-M_{10}^{2}}\right) & \cos \left(\frac{k L_{7}}{1-M_{10}^{2}}\right)
\end{array}\right]
$$

$$
\left(\begin{array}{c}
p_{11} \\
\rho_{o} c_{o} u_{11}
\end{array}\right)
$$

and the four-pole matrix between pt $13(z=0)$ and pt 14 $\left(z=L_{89}\right)$ with mean flow is expressed in equation (A18).

$$
\begin{gathered}
\left(\begin{array}{c}
p_{13} \\
\rho_{o} c_{o} u_{13}
\end{array}\right)=e^{-j \frac{M_{13} k L_{89}}{1-M_{13}^{2}}}\left[\begin{array}{cc}
\cos \left(\frac{k L_{89}}{1-M_{13}^{2}}\right) & j \sin \left(\frac{k L_{89}}{1-M_{13}^{2}}\right) \\
j \sin \left(\frac{k L_{89}}{1-M_{13}^{2}}\right) & \cos \left(\frac{k L_{89}}{1-M_{13}^{2}}\right)
\end{array}\right] \\
\left(\begin{array}{c}
p_{14} \\
\rho_{o} c_{o} u_{14}
\end{array}\right)
\end{gathered}
$$

\section{APPENDIX B}

\section{Transfer Matrix of Extended Duct}

The equation of mass continuity between pt 5 and pt 7 with mean flow is [8]

$$
\begin{aligned}
& c_{o} \rho_{0} S_{5} u_{5}+c_{o} \frac{s_{5} V_{5}}{c_{o}^{2}} p_{5} \\
& =c_{o} \rho_{o} S_{7} u_{7}+c_{o} \rho_{o} S_{6} u_{6} \\
& +c_{o} \frac{S_{7} V_{7}}{c_{o}^{2}}\left(p_{7}-\frac{p_{o}}{C_{v}} \frac{R k M_{7} Y_{7}}{p_{o}} \frac{v_{c, 7}-M_{7} p_{c, 7} / Y_{7}}{1-M_{7}^{2}}\right)
\end{aligned}
$$

A concept of static enthalpy deduced by Munjal [6] Sathyanarayana [7] is expressed as

$$
\left[\begin{array}{l}
p_{7} \\
u_{7}
\end{array}\right]=\left[\begin{array}{cc}
1 & -M_{7} Y_{7} \\
-\frac{M_{7}}{Y_{7}} & 1
\end{array}\right]\left[\begin{array}{l}
p_{c, 7} \\
v_{c, 7}
\end{array}\right]
$$

By substituting equation (B2) into equation (B1), one has

$$
\begin{aligned}
& c_{o} \rho_{o} S_{5} u_{5}+S_{5} M_{5} p_{5} \\
& =c_{o} \rho_{o} S_{7} u_{7}\left[1-\frac{(\gamma-1) k_{c}}{1-M_{7}^{2}}\right]+\left(M_{7} S_{7} p_{7}+c_{o} \rho_{o} u_{6} S_{6}\right)
\end{aligned}
$$

The equation of momentum for steady flow is

$$
\begin{aligned}
& S_{5} p_{5}+2 \rho_{o} S_{5} V_{5} u_{5}+\frac{S_{5} V_{5}^{2}}{c_{o}^{2}} p_{5} \\
& =-c_{1}\left(S_{7} p_{7}+2 \rho_{o} S_{7} V_{7} u_{7}+\frac{S_{7} V_{7}^{2}}{c_{o}^{2}}\left[\begin{array}{c}
p_{7}-(\gamma-1) k_{c} M_{7} Y_{7} \\
\frac{v_{c, 7}-M_{7} p_{c, 7} / Y_{7}}{1-M_{7}^{2}}
\end{array}\right]\right)
\end{aligned}
$$

$$
-c_{2} S_{6} p_{6}
$$

By substituting equation (B2) into equation (B4), one has

$$
\begin{aligned}
& S_{5}\left(1+M_{5}^{2}\right) p_{5}+2 \rho_{o} c_{o} S_{5} M_{5} u_{5}+c_{1}\left(S_{7}+S_{7} M_{7}^{2}\right) p_{7} \\
& =-c_{1}\left(2 S_{7} M_{7}+\frac{S_{7}(\gamma-1) k_{c} M_{7}^{3}}{1-M_{7}^{2}}\right) \rho_{o} c_{o} u_{7}-c_{2} S_{6} p_{6}
\end{aligned}
$$

The equation of energy conservation for steady flow is

$$
p_{5} \rho_{o} V_{5} u_{5}=p_{7}+\rho_{o} V_{7} u_{7}+k_{c} \rho_{o} V_{7} u_{7}
$$

With the rigid wall at boundary, one has

$$
\frac{p_{6}}{\rho_{o} c_{o} u_{6}}=-j \cot \left(k L_{4}\right)
$$

By taking equation (B7) into equations (B3), (B5), and (B6), the transfer matrix between pt 5 and pt 7 is thus illustrated as

$$
\left(\begin{array}{c}
p_{5} \\
\rho_{o} c_{o} u_{5}
\end{array}\right)=\left[\begin{array}{ll}
T R 4_{1,1} & T R 4_{1,2} \\
T R 4_{2,1} & T R 4_{2,2}
\end{array}\right]\left(\begin{array}{c}
p_{7} \\
\rho_{o} c_{o} u_{7}
\end{array}\right)
$$

As the derivation of Eq. (B8), the transfer matrix between pt 2 and pt 4 is expressed as

$$
\left(\begin{array}{c}
p_{2} \\
\rho_{o} c_{o} u_{2}
\end{array}\right)=\left[\begin{array}{ll}
T R 2_{1,1} & T R 2_{1,2} \\
T R 2_{2,1} & T R 2_{2,2}
\end{array}\right]\left(\begin{array}{c}
p_{4} \\
\rho_{o} c_{o} u_{4}
\end{array}\right)
$$


Similarly, the transfer matrix between pt 8 and pt 10 is expressed as

$$
\left(\begin{array}{c}
p_{8} \\
\rho_{o} c_{o} u_{8}
\end{array}\right)=\left[\begin{array}{ll}
T R 6_{1,1} & T R 6_{1,2} \\
T R 6_{2,1} & T R 6_{2,2}
\end{array}\right]\left(\begin{array}{c}
p_{10} \\
\rho_{o} c_{o} u_{10}
\end{array}\right)
$$

and the transfer matrix between pt 11 and pt 13 is expressed as

$$
\left(\begin{array}{c}
p_{11} \\
\rho_{o} c_{o} u_{11}
\end{array}\right)=\left[\begin{array}{ll}
T R 8_{1,1} & T R 8_{1,2} \\
T R 8_{2,1} & T R 8_{2,2}
\end{array}\right]\left(\begin{array}{c}
p_{13} \\
\rho_{o} c_{o} u_{13}
\end{array}\right)
$$

